\title{
Oral Submucous Firosis: An Update on Etiopathogenesis
}

\section{Shriya Khera ${ }^{1 *}$ and Sunita Gupta ${ }^{2}$}

${ }^{1}$ Senior Research Associate, Oral Medicine and Radiology, Maulana Azad Institute

of Dental Sciences, MAMC Campus, New Delhi, India

${ }^{2}$ Professor and HOD, Department of Oral Medicine and Radiology, Maulana Azad

Institute of Dental Sciences, New Delhi, India

*Corresponding Author: Shriya Khera, Senior Research Associate, Oral Medicine and Radiology, Maulana Azad Institute of Dental Sciences, MAMC Campus, New Delhi, India.
Received: October 14, 2021

Published: November 15, 2021

(C) All rights are reserved by Shriya Khera and Sunita Gupta.

\begin{abstract}
The theories related to development of oral submucous fibrosis (OSMF) has been multifactorial. Many etiological factors include the consumption of areca nut (supari), betel leaves (paan), tobacco and intake of spicy food which are commonly found in asian foods. Systemic causes like nutritional deficiency, genetic predisposition, autoimmunity, heat shock proteins have also been proposed as causative factors for OSMF. Albeit the extensive documented aetiological factors, the precise etiology is not yet known and no conclusive evidence has been linked to the development and progression of Oral Submucous Fibrosis. The present review aims to decipher the various etiological agents and decoding their role in pathogenesis and progression of Oral Submucous Fibrosis.
\end{abstract}

Keywords: Oral Submucous Fibrosis; Areca Nut; Pathogenesis; Review

\section{Introduction}

Oral Submucous Fibrosis is a chronic, progressive, potentially malignant condition of the oral cavity that predominantly affects people of South-East Asian origin [1]. It is characterized by a juxtaepithelial inflammatory reaction followed by fibroelastic change in the lamina propria and epithelial atrophy which leads to stiffness of the oral mucosa, trismus and inability to eat. It affects most parts of the oral cavity and may extend over time to include the pharynx and the upper third of esophagus [2].

Various etiological factors such as areca nut, capsaicin in chillies, micronutrient deficiency and autoimmune nature of the disease has also been proposed [3]. The present review article aims to understand the detailed etiopathogenesis of oral submucous fibrosis.

\section{Etiopathogenesis}

The most important etiological factor in OSMF is the use of areca nut. Areca nut contains arecoline, arecadine, guvacoline and guvacine which are responsible for fibroblast proliferation. With the addition of slaked lime $\left(\mathrm{Ca}(\mathrm{OH})_{2}\right)$, arecoline gets hydrolyzed to arecadine amplifying fibroblastic proliferation and increasing collagen formation [3].

Arecoline causes increased collagen synthesis by OSMF fibroblasts as compared to normal fibroblasts. This leads to clonal selection of a cell population in altered tissues under the influence of IL-1 which are released by inflammatory cells [5]. The presence offlavanoids such as tannins and catechins stabilise the structure of non soluble collagen (Type 3 collagen to highly resistant Type 1 collagen) and retards collagenase activity [5,6]. Arecoline pro- 
motes Cystatin C leading to inhibition of collagen proteinases causing stabilization of collagen fibrils [7,8] (Figure 1).

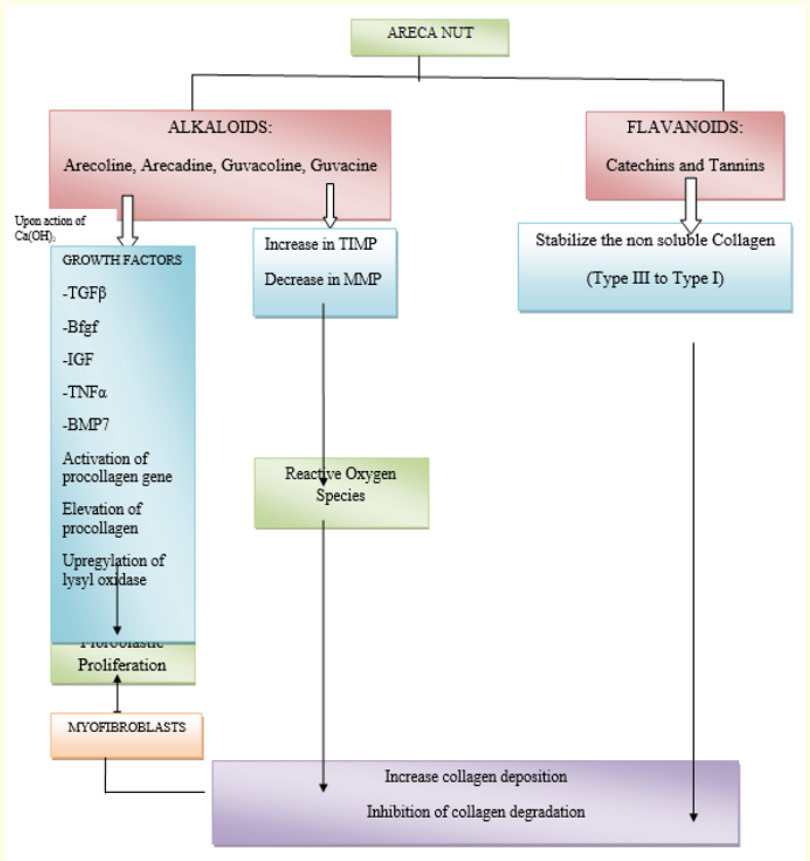

Figure 1: Role of areca nut in the pathogenesis of Oral Submucous Fibrosis.

\section{Role of copper}

Betel nut is usually grown at $15.5-38^{\circ} \mathrm{C}$ and is usually planted at pre monsoon period of May-June. The warm, moist and damp environment during this period predisposes various diseases. To avoid the onset of disease, a spray of $1 \%$ Bordeaux mixture in $10 \mathrm{~L}$ water is used as a prophylactic spray before the monsoons. Bordeaux mixture is a fungicide which is prepared by adding milk of lime to a solution of copper sulphate to withstand heavy rainfall [9]. The high concentration of copper in areca nut is due to praying of Bordeaux mixture that acts as a preservative [9].

On a molecular level, a significant amount of copper leads to up regulation of lysyl oxidase causing inhibition of collagenase degradation by activating tissue inhibitor of matrix metalloproteinase gene and plasminogen inhibitor activator gene leading to increase in production of insoluble collagen $[10,11]$ (Figure 2).

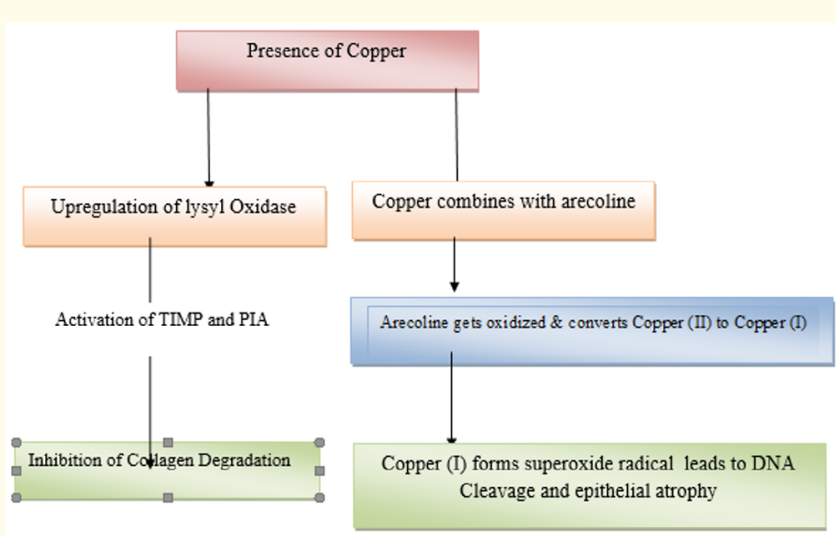

Figure 2: Role of copper in the pathogenesis of Oral Submucous Fibrosis.

\section{Role of immunity}

The normal collagen degradation is by phagocytosis which is inversely dose dependent to arecoline, safrole and nicotine in saliva. In OSMF, decrease in $\mathrm{T}$ cell activity leads to cell mediated immunity and causes impaired phagocytic activity [12]. Increased reactive oxygen species are formed by auto-oxidation in saliva or via intracellular metabolic activation [13].

Areca nut leads to upregulation of proinflammatory cytokines i.e. IL-6 and IL-8, TNF- $\alpha$, TGF- $\beta$, platelet-derived growth factor(PDGF), basic fibroblast growth factors and decreased expression of antifibrotic cytokine IF- $\gamma$ leading to increased fibrosis. This leads to altered immune response with increased Antigen Presenting Cells and lymphocytes and increase in permeability of arecoline and arecadine into the oral mucosa [14-16] (Figure 3).

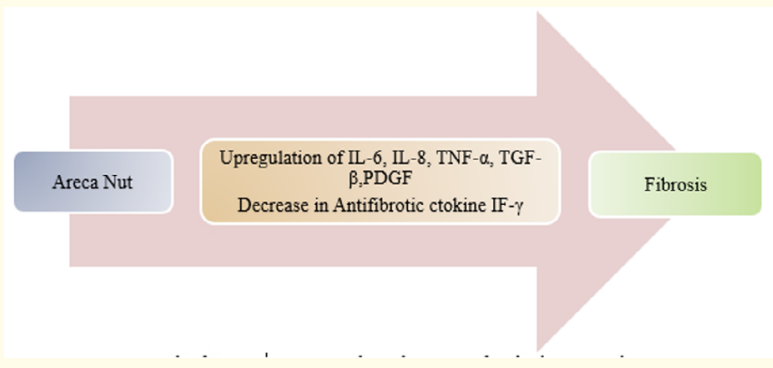

Figure 3: Role of immune response in the pathogenesis of Oral Submucous Fibrosis. 
An imbalance between the tissue inhibitors of matrix proteinases (TIMP) and matrix metalloproteinase's (MMP's) leads to increased deposition of Extracellular Matrix [17].

\section{Genetic susceptibility}

A strong genetic susceptibility leading to dysregulation of collagen metabolism causing highly resistant collagen and genes such as COL1A2, COL3A1, COL6A1, COL6A3 and COL7A1 are also linked to the progression of the disease [18].

\section{Autoimmunity}

A role of autoimmunity has also been seen as an etiological factor in the development and progression of the disease. Balram., et al. suggested that there is an increase in IgG, IgA and IgM and raised serum levels in patients with Oral Submucous Fibrosis [19,20].

Heat shock proteins

$47 \mathrm{kDa}$ Heat shock proteins are involved in the synthesis, processing and assembly of various collagen and is shown to cause accumulation of collagen in the mucosal connective tissue leading to accelerated fibroblastic activity [21,22].

\section{Nutritional deficiencies}

Deficiency of iron, vitamin and protein has been linked with the progression of OSMF [23]. Hydroxyproline is present in collagen as hydroxylated form (4 hydroxyl proline) with the use of iron and ascorbic acid. Deficiency of iron leads to decrease in vascularity due to reduction in cytochrome oxidase leading to atrophy of epithelium, causing burning sensation and ulcerations. A vicious cycle forms due to inability to consume food due to burning leading to anaemia $[24,25]$.

\section{Conclusion}

Regardless of the extensive studies that have been done on the role of various etiological agents and their etiopathogenesis on the development of Oral Submucous Fibrosis, it is still understood in a passable way. The present review aims to highlight and explain the various aetiologies and their pathogenesis in the progression of Oral Submucous Fibrosis to ameliorate the diagnosis and treatment of the affected patients.

\section{Bibliography}

1. Rajendran R. "Oral submucous fibrosis: etiology, pathogenesis and future research". Bulletin of World Health Organization 72.6 (1994): 985-996.

2. Pindborg J and Sirsat S. "Oral submucous fibrosis". Oral Surgery, Oral Medicine, Oral Pathology, and Oral Radiology 22.6 (1966): 764-779.

3. Tilakaratne WM., et al. "Oral submucous fibrosis: Review on aetiology and pathogenesis". Oral Oncology 42 (2006): 561568.

4. Salcedo R., et al. "Vascular endothelial growth factor and basic fibroblast growth factor induce expression of CXCR4 on human endothelial cells: in vivo neovascularization induced by stromal-derived factor-1alpha". The American Journal of $\mathrm{Pa}$ thology 154 (1999): 1125-1135.

5. Meghii S., et al. "An in vitro comparison of human fibroblasts from normal and oral submucous fibrosis tissue". Archives of Oral Biology 32 (1987): 213-215.

6. Utsunomiya H., et al. "Extracellular matrix remodeling in oral submucous fibrosis; its stage-specific modes revealed by immuno-histochemistry and in-situ hybridization". Journal of Oral Pathology and Medicine 34 (2005): 498-507.

7. Anila Namboodiripad PC. "Cystatin C. Its role in pathogenesis of OSMF". Journal of Oral Biology and Craniofacial Research 4.1 (2014): 42-46.

8. Arakeri G., et al. "Oral submucous fibrosis: An update on current theories of pathogenesis". Journal of Oral Pathology and Medicine 46.6 (2017): 406-412.

9. Nzegbule EC. "Assesment of copper levels in the soil and vegetation following repeated application of Bordeaux mixture to a cocoa plantation in south-eastern Nigeria”. Nigerian Agricultural Journal 34 (2003): 97-102.

10. Arakeri G and Brennan PA. "Oral submucous fibrosis: an overview of the etiology, pathogenesis, classification, and principles of management". British Journal of Oral and Maxillofacial Surgery 51 (2013): 587-593. 
11. Rajalalitha P and Vali S. "Molecular pathogenesis of oral submucous fibrosis- a collagen metabolic disorder". Journal of Oral Pathology and Medicine 34 (2005): 321-328.

12. Chang YC., et al. "Elevated vimentin expression in buccal mucosal fibroblasts by arecoline in vitro as a possible pathogenesis for oral submucous fibrosis". Oral Oncology 38 (2002): 425-430.

13. Shieh DH., et al. "Effects of arecoline, safrole, and nicotine on collagen phagocytosis by human buccal mucosal fibroblasts as a possible mechanism for oral submucous fibrosis in Taiwan. Journal of Oral Pathology and Medicine 33 (2004): 581-587.

14. Wang CC., et al. "Areca nut extract suppresses T-cell activation and interferon-c production via the induction of oxidative stress". Food and Chemical Toxicology 45 (2007): 1410-1418.

15. Jeng JH., et al. "Role of areca nut in betel quid-associated chemical carcinogenesis: current awareness and future perspectives". Oral Oncology 37 (2001): 477-492.

16. Aziz SR. "Oral submucous fibrosis: case report and review of diagnosis and treatment". Journal of Oral and Maxillofacial Surgery 66.11 (2008): 2386-2389.

17. Haque MF., et al. "Oral submucous fibrosis patients have altered levels of cytokine production". Journal of Oral Pathology and Medicine 29 (2000): 123-128.

18. Haque MF., et al. "Immunolocalization of cytokines and growth factors in oral submucous fibrosis". Cytokine 10 (1998): 713719.

19. De Waal J., et al. "The fibroblast population in Oral submucous fibrosis". Journal of Oral Pathology and Medicine 26 (1997): 6974.

20. Balaram P., et al. "Immunology of premalignant and malignant conditions of the oral cavity. Part II. Circulating immune complexes". Journal of Oral Pathology and Medicine 16 (1987): 389-391.

21. Remani P., et al. "Circulating immune complexes as an immunological marker in premalignant and malignant lesions of the oral cavity". Cancer Letters 40 (1988): 185-191.
22. Dyavanagoudar S N. "Oral Submucous Fibrosis: Review on Etiopathogenesis". Journal Cancer Science and Theraphy 1 (2009): 72-77.

23. Jayanthi V., et al. "Oral submucosal fibrosis a preventable disease". Gut 33 (1992): 4-6.

24. Khanna SS and Karjodkar FR. "Circulating Immune Complexes and trace elements (Copper, Iron and Selenium) as markers in oral precancer and cancer: A randomised, controlled clinical trial". Head and Face Medicine 2 (2006): 33.

25. Anuradha CD and Devi CS. "Serum protein, ascorbic acid and iron and tissue collagen in Oral submucous fibrosis. - a preliminary study". Indian Journal of Medical Research 98 (1993): 147-151.

26. Taneja L., et al. "Haemoglobin levels in patients with oral submucous fibrosis". Journal of Indian Academy of Oral Medicine and Radiology 19 (2007): 329-333.

\section{Volume 5 Issue 12 December 2021}

(C) All rights are reserved by Shriya Khera and Sunita Gupta. 